\title{
Quasi-Monte Carlo for finance beyond Black-Scholes
}

\author{
Jan Baldeaux ${ }^{1}$
}

(Received 9 August 2008; revised 18 May 2009)

\begin{abstract}
Quasi-Monte Carlo methods are used to approximate integrals of high dimensionality. However, if the problem under consideration is of unbounded dimensionality, it is not obvious if one can apply quasiMonte Carlo methods at all. We introduce a hybrid approach combining quasi-Monte Carlo and Monte Carlo methods and apply it to a finance problem of unbounded dimensionality. We find that this hybrid approach improves on a Monte Carlo approach.
\end{abstract}

\section{Contents}

1 Introduction

C885

2 Quasi-Monte Carlo point sets and the finance problem

C886

3 Monte Carlo approach to pricing lookback options in the Kou model

C888

http://anziamj . austms.org.au/ojs/index.php/ANZIAMJ/article/view/1407 gives this article, (c) Austral. Mathematical Soc. 2009. Published May 27, 2009. ISSN 1446-8735. (Print two pages per sheet of paper.) 
4 Hybrid approach to pricing lookback options in the Kou model

C891

5 Numerical results and conclusion

C895

References

C896

\section{Introduction}

Quasi-Monte Carlo (QMC) methods are techniques for high dimensional numerical integration. The pricing of financial derivatives in Gaussian models results in integration problems to which QMC methods are routinely applied and significant reductions in computational effort have been observed $[1$, e.g.]. However, the shortcomings of Gaussian models are well-known, and from a finance point of view, the usage of jump diffusion models such as the Kou model [4, e.g.] might be more appropriate. Yet, the pricing of financial derivatives in the Kou model can result in problems of unbounded dimensionality, and it is not obvious if QMC methods can be applied at all.

We show how to formulate the finance problem in such a way that QMC methods can be applied. This allows us to enjoy the benefits associated with QMC methods but also the advantages of using a model that is more sophisticated than a Gaussian model.

Having recalled properties of QMC point sets and the Kou model and defined lookback options in Section 2, Section 3 shows why the pricing of lookback options in the Kou model can result in an integration problem of unbounded dimensionality. Section 4 recalls stratification, a variance reduction technique, and uses it to construct a hybrid approach combining QMC and Monte Carlo (MC) methods. Section 5 presents numerical results showing that the hybrid approach outperforms the standard Monte Carlo approach. 


\section{Quasi-Monte Carlo point sets and the finance problem}

We firstly recall some basic properties of QMC point sets and then introduce the finance problem.

QMC methods are equal weight integration formulas to estimate high dimensional integrals over the unit cube. In mapping the integration problem to the unit cube, we often need to invert probability distributions and we hence recall the well-known generalised inverse function, for example Glasserman [3, p.55]: if $\mathrm{F} \sim \mathrm{F}$, then the generalised inverse of $\mathrm{F}, \mathrm{F}^{-1}(\mathrm{u})$, is

$$
\mathrm{F}^{-1}(u)=\inf \{z \in \mathbb{R}: F(z) \geqslant u\}, \quad u \in[0,1] .
$$

We point out that all generalised inverse functions needed for this paper are available in standard computer packages such as MATLAB. Regarding the QMC point sets, we make use of digital nets [6, e.g.] and randomize these using a digital b-ary shift [6, e.g.] to compute standard errors.

Turning to the finance problem, we firstly recall properties of the Kou model [4, e.g.]. We assume that we deal with a probability space $(\Omega, \mathcal{F}, \operatorname{Pr})$ on which we introduce the stochastic processes $\left(Z_{t}\right)_{t \geqslant 0}$ and $\left(S_{t}\right)_{t \geqslant 0}$ in Definition 1. The following density function is used in Definition 1 :

$$
f(x)=p \eta^{+} \exp \left(-\eta^{+} x\right) \mathbb{I}_{x \geqslant 0}+(1-p) \eta^{-} \exp \left(\eta^{-} x\right) \mathbb{I}_{x<0}, \quad \text { for all } x \in \mathbb{R},
$$

where $0 \leqslant p \leqslant 1, \eta^{+}>1, \eta^{-}>0$. The cumulative distribution corresponding to density $f$, denoted by $F$, is invertible and we denote its inverse by $F^{-1}(u), u \in[0,1]$. The inverse is computed explicitly as

$$
\mathrm{F}^{-1}(\mathfrak{u})=\frac{\ln (u)-\ln (1-p)}{\eta^{-}} \mathbb{I}_{\mathfrak{u} \leqslant 1-p}+\frac{\ln (1-u)-\ln (p)}{-\eta^{+}} \mathbb{I}_{\mathfrak{u}>1-p}, \quad u \in[0,1] .
$$

We also set $\mu=r-\frac{1}{2} \sigma_{S}^{2}-\lambda \int_{\mathbb{R}}(\exp (x)-1) f(x) d x$, for $\sigma_{S} \in \mathbb{R}^{+}, r \in \mathbb{R}^{+}$. 
Definition 1 Let $\left(\mathrm{N}_{\mathrm{t}}\right)_{\mathrm{t} \geqslant 0}$ be a Poisson process with intensity $\lambda>0$ and jump times $\left(\tau_{i}\right)_{i=1}^{N_{t}},\left(W_{t}\right)_{t \geqslant 0}$ a Brownian motion and $\left(\mathrm{Y}_{\mathrm{k}}\right)_{\mathrm{k} \geqslant 1}$ independent, identically distributed random variables with distribution $\mathrm{F}$. Then

$$
Z_{t}=\mu t+\sigma_{S} W_{t}+\sum_{k=1}^{N_{t}} Y_{k}
$$

and, for $\mathrm{S}_{0} \in \mathbb{R}^{+}$,

$$
S_{t}=S_{0} \exp \left(Z_{t}\right)
$$

Of course $\left(S_{t}\right)_{t \geqslant 0}$ is the stock price process. We now focus on the financial derivative relevant for this article. We deal with a particular lookback option, a continuously monitored lookback put option with floating strike price, from now on referred to as the lookback option. Its pay-off at time $T$ is

$$
\max \left(B, \max _{0 \leqslant t \leqslant T} S_{t}\right)-S_{T}
$$

where $\mathrm{B} \in \mathbb{R}^{+}, \mathrm{B}>\mathrm{S}_{0}, \mathrm{~T} \in \mathbb{R}^{+}$, and its price by

$$
\mathbb{E}\left[e^{-r \mathrm{~T}}\left(\max \left(B, \max _{0 \leqslant t \leqslant T} S_{t}\right)-S_{T}\right)\right]=\mathbb{E}\left[e^{-r T} \max \left(B, \max _{0 \leqslant t \leqslant T} S_{t}\right)\right]-S_{0} .
$$

So the integration problem is reduced to approximating

$$
\mathbb{E}\left[\exp (-r T) \max \left(B, \max _{0 \leqslant t \leqslant T} S_{t}\right)\right]
$$

and we denote

$$
\mathrm{V}=\exp (-\mathrm{rT}) \max \left(\mathrm{B}, \max _{0 \leqslant \mathrm{t} \leqslant \mathrm{T}} \mathrm{S}_{\mathrm{t}}\right) ;
$$

being constant, $\exp (-\mathrm{rT})$ does not contribute to the computational performance though. We now show how to estimate prices of lookback options, assuming that the stock price is (3). 


\section{Monte Carlo approach to pricing lookback options in the Kou model}

We show how to price a lookback option in the Kou model using a Monte Carlo approach. This seems new, most likely due to the closed form solutions for prices of lookback options in the Kou model that are available in terms of special functions [4]. However, the methodology presented in this section is not limited to the Kou model: instead of assuming that jumps follow (1), any distribution that can be simulated can be used. We make the convention that $\tau_{0}=0$ and $\tau_{N_{T}+1}=T$.

We recall the definition (5) of $\mathrm{V}$ and denote independent, identically distributed copies of $V$ by $V_{i}, i=1, \ldots, n_{\mathrm{MC}}, n_{\mathrm{MC}} \in \mathbb{Z}^{+}$, so $n_{\mathrm{MC}}$ corresponds to the number of function evaluations for the naive $\mathrm{MC}$ approach. Then

$$
\mathrm{I}_{\mathrm{MC}}=\frac{1}{\mathrm{n}_{\mathrm{MC}}} \sum_{i=1}^{\mathrm{n}_{\mathrm{MC}}} \mathrm{V}_{\mathrm{i}}
$$

is an unbiased estimator of $\mathbb{E}[\mathrm{V}]$ and an unbiased estimator of its variance is

$$
\sigma_{\mathrm{MC}}^{2}=\frac{1}{n_{\mathrm{MC}}\left(n_{\mathrm{MC}}-1\right)} \sum_{i=1}^{n_{\mathrm{MC}}}\left(V_{i}-I_{\mathrm{MC}}\right)^{2} .
$$

However, the simulation of $V_{i}, i=1, \ldots, n_{\mathrm{MC}}$, is not immediately obvious and is explained below. The following notation is introduced:

$$
M_{l}=\max _{\tau_{l-1} \leqslant t<\tau_{l}} S_{t}, \quad l=1, \ldots, N_{T}+1,
$$

and we define the $\sigma$-algebra

$$
\mathcal{F}^{*}=\sigma\left(\left(\mathrm{N}_{\mathrm{t}}\right)_{\mathrm{t} \in[0, \mathrm{~T}]},\left(\mathrm{Y}_{\mathrm{l}}\right)_{l=1}^{\mathrm{N}_{\mathrm{T}}},\left(\mathrm{W}_{\tau_{\mathrm{l}}}\right)_{l=1}^{\mathrm{N}_{\mathrm{T}}}, \mathrm{W}_{\mathrm{T}}\right),
$$

that is the smallest $\sigma$-algebra with respect to which $\left(N_{t}\right)_{t \in[0, T]},\left(Y_{l}\right)_{l=1}^{N_{T}}$, $\left(W_{\tau_{l}}\right)_{l=1}^{N_{T}}$ and $W_{\mathrm{T}}$ are measurable. We express

$$
\mathbb{E}[\mathrm{V}]=\mathbb{E}\left\{\mathbb{E}\left[\exp (-\mathrm{rT}) \max \left(\mathrm{B}, \max _{\mathrm{l}=1, \ldots, \mathrm{N}_{\mathrm{T}}+1} \mathrm{M}_{\mathrm{l}}\right) \mid \mathcal{F}^{*}\right]\right]
$$


The equality (8) suggests that one can simulate $V$ by simulating $\left(M_{l}\right)_{l=1}^{N_{T}+1}$. To be able to generate the $M_{l}$, we need to know their distributions and be able to invert these. This is addressed in the next lemma, parts 2 and 3, where we set $S_{\tau_{l}^{-}}=S_{l^{-}}$and $S_{\tau_{l-1}}=S_{l-1}, l=1, \ldots, N_{T}+1$.

Lemma 2 The following properties hold

1. Conditional on $\mathcal{F}^{*}$, the $\left(\mathrm{M}_{\mathrm{l}}\right)_{\mathrm{l}=1}^{\mathrm{N}_{\mathrm{T}}+1}$ are independent.

2. The distribution of $\mathrm{M}_{\mathrm{l}}$, conditional on $\mathcal{F}^{*}, \mathrm{~F}_{\mathrm{M}_{\mathrm{l}}}$, is

$$
\begin{aligned}
& \mathrm{F}_{M_{l}}(\mathrm{~b})=\operatorname{Pr}\left(M_{\mathrm{l}} \leqslant \mathrm{b} \mid \mathcal{F}^{*}\right) \\
& =\left(1-\exp \left(-\frac{2\left(\ln \left(S_{l^{-}}\right)-\ln (\mathrm{b})\right)\left(\ln \left(S_{l-1}\right)-\ln (\mathrm{b})\right)}{\left(\tau_{l}-\tau_{l-1}\right) \sigma_{S}^{2}}\right)\right) \mathbb{I}_{\mathrm{b} \geqslant \max \left(S_{l^{-}}, S_{l_{-1}}\right)} .
\end{aligned}
$$

3. The inverse of $\mathrm{F}_{\mathrm{M}_{\mathrm{l}}}, \mathrm{F}_{\mathrm{M}_{\mathrm{l}}}^{-1}$, is

$$
\begin{aligned}
& \ln \left(F_{M_{l}}^{-1}(u)\right) \\
& =\frac{\ln \left(S_{l^{-}} S_{l-1}\right)+\sqrt{\left(\ln \left(S_{l^{-}}\right)-\ln \left(S_{l-1}\right)\right)^{2}-2\left(\tau_{l}-\tau_{l-1}\right) \sigma_{S}^{2} \ln (1-u)}}{2} .
\end{aligned}
$$

Proof: Parts 1 and 2 are given by Cont and Tankov [2, p.177]; Part 3 follows from Part 2.

Next, recall that if $\mathbf{Y}$ follows the Beta distribution with parameters $a$ and $b$, $\mathrm{Y} \sim \operatorname{Beta}(\boldsymbol{a}, \boldsymbol{b})$, then its density is

$$
f(x)=\frac{\Gamma(a+b)}{\Gamma(a) \Gamma(b)} x^{a-1}(1-x)^{b-1}, \quad 0 \leqslant x \leqslant 1,
$$

and the inverse of its distribution is denoted by $\mathrm{F}_{\text {Beta }}^{-1}(\mathrm{u}, \mathrm{a}, \mathrm{b})$. Regarding notation, if $X$ is normal with mean $\mu$ and variance $\sigma^{2}$, we write $X \sim N\left(\mu, \sigma^{2}\right)$, and the inverse of its distribution is denoted by $F_{N}^{-1}\left(u, \mu, \sigma^{2}\right)$. If $Y$ is Poisson with rate $\lambda$, we write $Y \sim P(\lambda)$, and the inverse of its distribution is denoted 
by $\mathrm{F}_{\mathrm{P}}^{-1}(\mathrm{u}, \lambda)$. Finally we state the following lemma (for example, Ross $[7$, Theorem 5.2 and Example 2.38]).

Lemma 3 Conditional on $\mathrm{N}_{\mathrm{T}}=\mathrm{n}, \tau_{\mathrm{l}}=\mathrm{a}, \tau_{\mathrm{m}}=\mathrm{b}, 0 \leqslant \mathrm{a}<\mathrm{b} \leqslant \mathrm{T}$ and $0 \leqslant \mathrm{l}<\mathrm{m} \leqslant \mathrm{n}+1$, the law of $\left(\tau_{\mathrm{l}+\mathrm{o}}\right)_{\mathrm{o}=1}^{\mathrm{m}-\mathrm{l}-1}$ is

$$
\frac{\tau_{l+o}-\tau_{l}}{\tau_{m}-\tau_{l}} \sim \operatorname{Beta}(o, m-l-o), \quad o=1, \ldots, m-l-1 .
$$

We are now in a position to state the algorithm showing how to obtain one realisation of $\mathrm{V}$, which is needed for the $\left(\mathrm{V}_{i}\right)_{i=1}^{\mathfrak{n}_{\mathrm{MC}}}$ in (6).

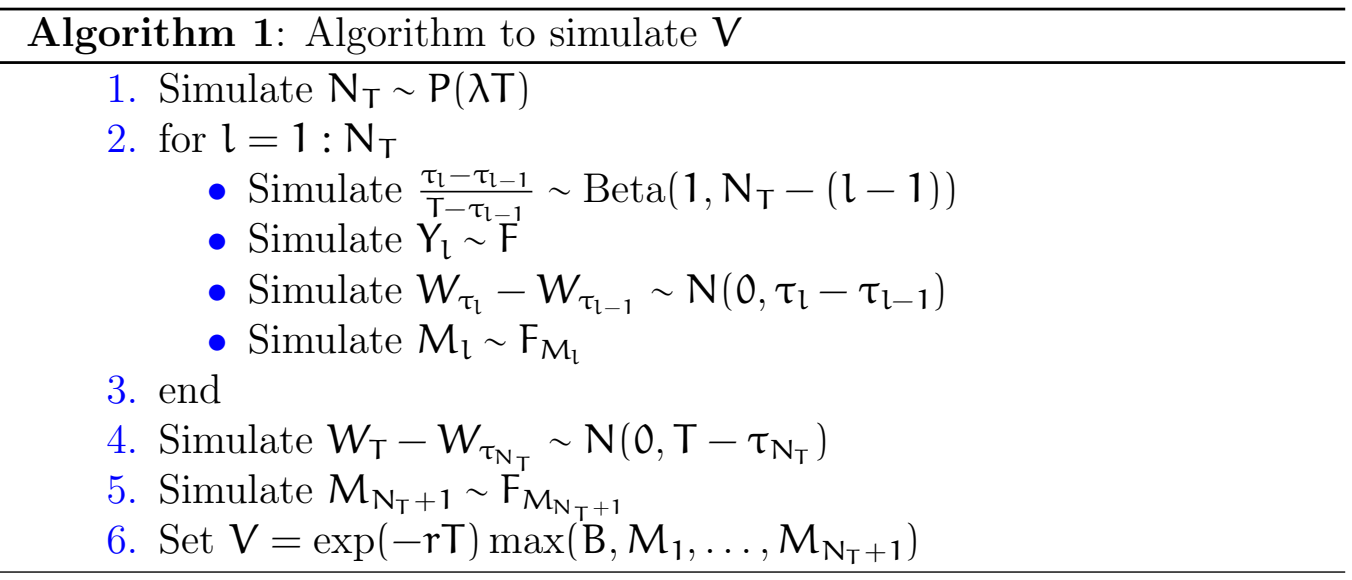

The following remark shows that for purposes of QMC, Algorithm 1 is not useful.

Remark 4 We note that the formulation of the problem provided by Algorithm 1 is not suitable for QMC methods. This is because $N_{T}$ is not bounded resulting in a problem whose dimension is very high, in principle infinite. Furthermore, dealing with a QMC point set, a particular dimension should correspond always to the same random variable, else the favourable uniformity properties of the QMC point set are not preserved. Algorithm 1 does not ensure this. 


\section{Hybrid approach to pricing lookback options in the Kou model}

We show how to price lookback options in the Kou model by combining QMC and MC methods, a hybrid approach. The hybrid approach is based on stratification - a well-known variance reduction technique described, for example, by Glasserman [3], Stratification is a technique constraining the fraction of observations drawn from specific subsets (strata) of the sample space. For the purposes of our problem, we fix $k^{*} \in \mathbb{Z}^{+}$and choose the following $k^{*}+2$ strata: $\left\{N_{T}=k_{\}_{k=0}}^{k^{*}}\right.$ and $\left\{N_{T}>k^{*}\right\}$. Consequently, we rewrite

$$
\mathbb{E}[\mathrm{V}]=\sum_{\mathrm{k}=0}^{\mathrm{k}^{*}} \mathbb{E}\left[\mathrm{V} \mid \mathrm{N}_{\mathrm{T}}=\mathrm{k}\right] \operatorname{Pr}\left(\mathrm{N}_{\mathrm{T}}=\mathrm{k}\right)+\mathbb{E}\left[\mathrm{V} \mid \mathrm{N}_{\mathrm{T}}>\mathrm{k}^{*}\right] \operatorname{Pr}\left(\mathrm{N}_{\mathrm{T}}>\mathrm{k}^{*}\right)
$$

We note though that using Black-Scholes arguments, we arrive at a closed form solution for $\mathbb{E}\left[V \mid N_{T}=0\right]$, denoted by $I_{0}$, hence there is no need to estimate $\mathbb{E}\left[V \mid N_{T}=0\right]$. In addition, we set $p_{k}=\operatorname{Pr}\left(N_{T}=k\right), k=0, \ldots, k^{*}$ and $p_{k^{*}+1}=\operatorname{Pr}\left(N_{T}>k^{*}\right)$ and define the following random variables, where $\mathcal{B}(\mathbb{R})$ denotes the Borel $\sigma$-algebra on $\mathbb{R}$

Definition 5 Let $\mathrm{k}^{*} \in \mathbb{Z}^{+}$and $\mathrm{S} \in \mathcal{B}(\mathbb{R})$, then $\tilde{\mathrm{V}}_{\mathrm{k}}, \mathrm{k}=0, \ldots, \mathrm{k}^{*}+1$ are random variables with respective laws

$$
\operatorname{Pr}\left(\tilde{\mathrm{V}}_{\mathrm{k}} \in \mathrm{S}\right)=\operatorname{Pr}\left(\mathrm{V} \in \mathrm{S} \mid \mathrm{N}_{\mathrm{T}}=\mathrm{k}\right), \quad \mathrm{k}=0, \ldots, \mathrm{k}^{*}
$$

and

$$
\operatorname{Pr}\left(\tilde{\mathrm{V}}_{\mathrm{k}^{*}+1} \in \mathrm{S}\right)=\operatorname{Pr}\left(\mathrm{V} \in \mathrm{S} \mid \mathrm{N}_{\mathrm{T}}>\mathrm{k}^{*}\right)
$$

With this definition, (10) becomes

$$
\mathbb{E}[\mathrm{V}]=\sum_{\mathrm{k}=0}^{\mathrm{k}^{*}+1} \mathbb{E}\left[\tilde{\mathrm{V}}_{\mathrm{k}}\right] \mathrm{p}_{\mathrm{k}}
$$


Recall Remark 4, stating that the naive MC approach is not suitable for QMC methods, as it results in problems of unbounded dimensionality and the allocation of variables to dimensions is not fixed. However, considering formulation (11), the $\mathbb{E}\left[\tilde{V}_{k}\right], k=1, \ldots, k^{*}$, are each of fixed dimension and it is possible to allocate a particular dimension to a particular random variable. Consequently, we formulate $\mathbb{E}\left[\tilde{V}_{k}\right], k=1, \ldots, k^{*}$, as integration problems and apply QMC methods to them. Of course $\mathbb{E}\left[\tilde{V}_{k^{*}+1}\right]$ is still of unbounded dimension and it is not possible to allocate a particular dimension to a particular random variable, so $\mathbb{E}\left[\tilde{\mathrm{V}}_{\mathrm{k}^{*}+1}\right]$ cannot be estimated using QMC methods. Instead MC methods will still be used. We now state the integration problem for $k=1$, but the same reasoning holds for $k=2, \ldots, k^{*}$.

$$
\begin{aligned}
& \mathbb{E}\left[\tilde{V}_{1}\right]=\mathbb{E}\left[\tilde{V}_{1}\left(\tau_{1}, \mathrm{Y}_{1}, W_{\tau_{1}}, M_{1}, W_{T}, M_{2}, 1\right)\right] \\
= & \int_{u_{1}=0}^{1} \ldots \int_{u_{6}=0}^{1} \tilde{V}_{1}\left\{T_{\text {Beta }}^{-1}\left(u_{1}, 1,1\right), F_{J}^{-1}\left(u_{2}\right),\right. \\
& F_{N}^{-1}\left[u_{3}, 0, T_{\text {Beta }}^{-1}\left(u_{1}, 1,1\right)\right], F_{M_{1}}^{-1}\left(u_{4}, u_{1}, u_{3}\right), F_{N}^{-1}\left[u_{3}, 0, T_{\text {Beta }}^{-1}\left(u_{1}, 1,1\right)\right] \\
& \left.+F_{N}^{-1}\left[u_{5}, 0, T-T_{\text {Beta }}^{-1}\left(u_{1}, 1,1\right)\right], F_{M_{2}}^{-1}\left(u_{6}, u_{1}, u_{2}, u_{3}, u_{5}\right)\right\} d u_{6} \ldots d u_{1}
\end{aligned}
$$

where $\mathrm{F}_{\mathbf{M}_{1}}^{-1}\left(\mathfrak{u}_{4}, \mathfrak{u}_{1}, \mathfrak{u}_{3}\right)=\exp \left[\mathbf{a}\left(\mathfrak{u}_{4}, \mathfrak{u}_{1}, \mathfrak{u}_{3}\right)\right]$ and

$a\left(u_{4}, u_{1}, u_{3}\right)$

$=\frac{\ln \left[S_{1^{-}}\left(u_{1}, u_{3}\right) S_{0}\right]+\sqrt{\left[\ln \left(S_{1^{-}}\left(u_{1}, u_{3}\right)\right)-\ln \left(S_{0}\right)\right]^{2}-2 \tau_{1}\left(u_{1}\right) \sigma_{S}^{2} \ln \left(1-u_{4}\right)}}{2}$

where $S_{1^{-}}\left(u_{1}, u_{3}\right)=S_{0} \exp \left\{\mu T_{\text {Beta }}^{-1}\left(u_{1}, 1,1\right)+\sigma_{S} F_{N}^{-1}\left[u_{3}, 0, T_{\text {Beta }}^{-1}\left(u_{1}, 1,1\right)\right]\right\}$ and $\tau_{1}\left(u_{1}\right)=T_{\text {Beta }}^{-1}\left(u_{1}, 1,1\right)$. The formula for $F_{M_{2}}^{-1}\left(u_{6}, u_{1}, u_{2}, u_{3}, u_{5}\right)$ can be derived using similar arguments.

Given a digital net in base $b,\left(u_{i}\right)_{i=1}^{n}, u_{i} \in[0,1]^{6}$, and $q$ independent random vectors $\Delta_{j}, j=1, \ldots, q$ uniformly distributed in $[0,1]^{6}$, we randomize $\left(u_{i}\right)_{i=1}^{n}$ using a digital b-ary shift $[6$, e.g.], where $\oplus$ means that for each dimension, 
we perform the digit-wise addition modulo $b$.

$$
\begin{aligned}
\mathbb{E}\left[\tilde{V}_{1}\right] \approx & \frac{1}{q} \sum_{j=1}^{\mathrm{q}} \frac{1}{\mathrm{n}} \sum_{i=1}^{\mathrm{n}} \tilde{\mathrm{V}}_{1}\left\{\mathrm{TF}_{\text {Beta }}^{-1}\left(\mathrm{u}_{\mathrm{i} 1} \oplus \Delta_{\mathrm{j} 1}, 1,1\right), \mathrm{F}_{\mathrm{J}}^{-1}\left(\mathrm{u}_{\mathrm{i} 2} \oplus \Delta_{\mathrm{j} 2}\right),\right. \\
& \mathrm{F}_{\mathrm{N}}^{-1}\left(\mathrm{u}_{\mathrm{i} 3} \oplus \Delta_{\mathrm{j} 3}, 0, \mathrm{TF}_{\text {Beta }}^{-1}\left(\mathrm{u}_{\mathrm{i} 1} \oplus \Delta_{\mathrm{j} 1}, 1,1\right)\right), \\
& \mathrm{F}_{\mathrm{M}_{1}}^{-1}\left(\mathrm{u}_{\mathrm{i} 4} \oplus \Delta_{\mathrm{j} 4}, \mathrm{u}_{\mathrm{i} 1} \oplus \Delta_{\mathrm{j} 1}, \mathrm{u}_{\mathrm{i} 3} \oplus \Delta_{\mathrm{j} 3}\right), \\
& \mathrm{F}_{\mathrm{N}}^{-1}\left(\mathrm{u}_{\mathrm{i} 3} \oplus \Delta_{\mathrm{j} 3}, 0, \mathrm{TF}_{\text {Beta }}^{-1}\left(\mathrm{u}_{\mathrm{i} 1} \oplus \Delta_{\mathrm{j} 1}, 1,1\right)\right) \\
& +\mathrm{F}_{\mathrm{N}}^{-1}\left(\mathrm{u}_{\mathrm{i} 4} \oplus \Delta_{\mathrm{j} 4}, 0, \mathrm{~T}-\mathrm{TF}_{\text {Beta }}^{-1}\left(\mathrm{u}_{\mathrm{i} 1} \oplus \Delta_{\mathrm{j} 1}, 1,1\right)\right), \\
& \left.\mathrm{F}_{\mathrm{M}_{2}}^{-1}\left(\mathrm{u}_{\mathrm{i} 6} \oplus \Delta_{\mathrm{j} 6}, \mathrm{u}_{\mathrm{i} 1} \oplus \Delta_{\mathrm{j} 1}, \mathrm{u}_{\mathrm{i} 2} \oplus \Delta_{\mathrm{j} 2}, \mathrm{u}_{\mathrm{i} 3} \oplus \Delta_{\mathrm{j} 3}, \mathrm{u}_{\mathrm{i} 5} \oplus \Delta_{\mathrm{j} 5}\right)\right\} .
\end{aligned}
$$

We hence get the following unbiased estimator for

$$
\mathbb{E}\left[\tilde{\mathrm{V}}_{1}\right] \approx \frac{1}{\mathrm{q}} \sum_{j=1}^{\mathrm{q}} \frac{1}{\mathrm{n}} \sum_{i=1}^{\mathrm{n}} \tilde{\mathrm{V}}_{1}\left(\mathrm{u}_{\mathrm{i}} \oplus \Delta_{\mathrm{j}}\right)=\mathrm{I}_{1} .
$$

Generalising this argument and using mutually independent $\Delta_{j}^{k}, j=1, \ldots, q$, $k=1, \ldots, k^{*}$, we obtain

$$
I_{k}=\frac{1}{q} \sum_{j=1}^{q} I_{k j}=\frac{1}{q} \sum_{j=1}^{q} \frac{1}{n_{k}} \sum_{i=1}^{n_{k}} \tilde{V}_{k}\left(u_{i} \oplus \Delta_{j}^{k}\right), \quad k=1, \ldots, k^{*},
$$

which are unbiased estimators for $\mathbb{E}\left[\tilde{\mathrm{V}}_{\mathrm{k}}\right]$ and we define the hybrid estimator

$$
\mathrm{I}_{\mathrm{HYB}}=\sum_{k=0}^{k^{*}+1} \mathrm{I}_{k} p_{k}
$$

As $\mathbb{E}\left[\tilde{\mathrm{V}}_{\mathrm{k}^{*}+1}\right]$ is estimated using MC methods, $\mathrm{I}_{\mathrm{k}^{*}+1}$ is defined via

$$
I_{k^{*}+1}=\frac{1}{n_{k^{*}+1}} \sum_{i=1}^{n_{k^{*}+1}} \tilde{V}_{k^{*}+1, i}
$$


The simulation of $\tilde{V}_{k^{*}+1}$ is detailed below. We now comment on the choice of the $n_{k}, k=1, \ldots, k^{*}$. We fix the total number of function evaluations for the hybrid approach to be $q \times n$ and set $n_{k}=\left\lfloor p_{k} n\right\rfloor, k=1, \ldots, k^{*}$. The case $\mathrm{I}_{\mathrm{k}^{*}+1}$ is treated separately, as $\mathbb{E}\left[\tilde{\mathrm{V}}_{\mathrm{k}^{*}+1}\right]$ is estimated via MC methods: We set $n_{k^{*}+1}$, the total number of function evaluations to be used for $I_{k^{*}+1}$, to be $n_{k^{*}+1}=n q-q \sum_{k=1}^{k^{*}} n_{k}$. Of course $I_{H Y B}$ is an unbiased estimator of $\mathbb{E}[\mathrm{V}]$ and by the independence of the random variables $\Delta_{\mathfrak{j}}^{k}, \mathfrak{j}=1, \ldots, \mathrm{q}$, $k=1, \ldots, k^{*}$, it follows that the variance of $I_{\mathrm{HYB}}$ is

$$
\operatorname{Var}\left(\mathrm{I}_{\mathrm{HYB}}\right)=\sum_{k=1}^{k^{*}+1} \operatorname{Var}\left(\mathrm{I}_{\mathrm{k}}\right) p_{\mathrm{k}}^{2}
$$

and an unbiased estimator of $\operatorname{Var}\left(\mathrm{I}_{\mathrm{HYB}}\right)$ is

$$
\sigma_{\mathrm{HYB}}^{2}=\sum_{k=1}^{k^{*}}\left[\frac{1}{q(q-1)} \sum_{j=1}^{q}\left(I_{k j}-I_{k}\right)^{2}\right] p_{k}^{2}+\sigma_{k^{*}+1}^{2} p_{k^{*}+1}^{2}
$$

where

$$
\sigma_{k^{*}+1}^{2}=\frac{1}{n_{k^{*}+1}\left(n_{k^{*}+1}-1\right)} \sum_{i=1}^{n_{k^{*}+1}}\left(I_{k^{*}+1}-\tilde{V}_{k^{*}+1, i}\right)^{2},
$$

with $n_{k^{*}+1}=n q-q \sum_{k=1}^{k^{*}} n_{k}$. Computing $I_{H Y B}$, we need to be able to obtain $\tilde{V}_{k}(u \oplus \Delta), k=1, \ldots, k^{*}$, for a QMC point $u \in[0,1]^{4 k+2}$ and $\Delta$ uniformly distributed in $[0,1]^{4 k+2}$, which is shown in Algorithm 2.

For $I_{k^{*}+1}$, which is estimated using MC methods, we make the following minor modification to Algorithm 1 to simulate $\tilde{V}_{k^{*}+1}$ : we replace step 1 by "Simulate $\mathrm{N}_{\mathrm{T}}$ conditional on $\mathrm{N}_{\mathrm{T}}>\mathrm{k}^{*}$ ", which requires us to simulate from conditional distributions, for example Glasserman [3, p.57], and leave the rest of Algorithm 1 unchanged. 


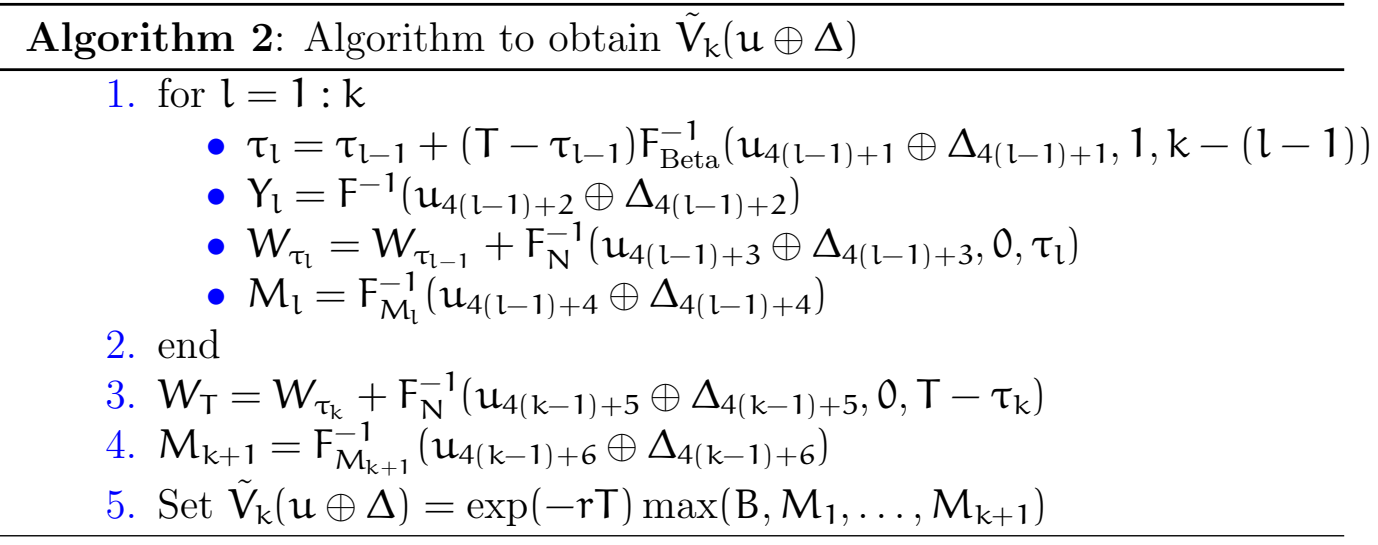

\section{$5 \quad$ Numerical results and conclusion}

We compare the approaches introduced in Sections 3 and 4. The following set of parameters is taken from Kou and Wang [4]. We set $B=110, S_{0}=100$, $\mathrm{T}=1$ and $r=0.05, \lambda=3, \sigma_{S}=0.2, p=0.3, \eta^{+}=50$ and $\eta^{-}=25$ in (2). For the hybrid approach, we need to choose $k^{*}$. We base our choices on the expected number of jumps on $[0, T]$, three in this case, and choose $k_{1}^{*}=4$ and $k_{2}^{*}=8$. The results presented in Table 1 were achieved as follows. For the hybrid approach (HYB), we vary $\mathrm{n}$ as shown in the table and set $\mathbf{q}=30$ in (12). To ensure the same numbers of function evaluations are used for both approaches, we set $n_{M C}=q \times n$ for the Monte Carlo approach (MC). We choose Sobol points, a digital net in base two, as our QMC point set. Standard errors were computed using (7) and (12), results for $k_{2}^{*}=8$ are given in brackets. Table 1 shows that the hybrid approach clearly outperforms the Monte Carlo approach and that increasing $k^{*}$ results in a decrease in standard errors, and seemingly an improved rate of convergence.

In conclusion, the hybrid approach allows us to apply QMC methods to the pricing of financial derivatives in the Kou model. This means that we can reduce the computational effort associated with a sophisticated model, an important consideration for practitioners. Looking ahead, many other mod- 


\begin{tabular}{lllll}
\hline & $n=256$ & $n=1024$ & $n=4096$ & $n=16384$ \\
\hline MC & 0.1713 & 0.0868 & 0.0433 & 0.0217 \\
HYB & $0.0970(0.0916)$ & $0.0402(0.0346)$ & $0.0169(0.0106)$ & $0.0083(0.0047)$ \\
\hline
\end{tabular}

TABLE 1: Standard errors for the MC point set based on $q \times n$ points and the QMC point set based on $n$ points, $q$ random shifts and $k_{1}^{*}=4\left(k_{2}^{*}=8\right)$.

els, arguably more sophisticated than the Kou model, have appeared in the literature [5, e.g.]. Studying how to reduce the associated computational effort using QMC methods is an exciting area of future research.

Acknowledgments The author gratefully acknowledges the support of the Australian Research Council Centre for Mathematics and Statistics of Complex Systems.

\section{References}

[1] R. E. Caflisch, W. J. Morokoff, and A. B. Owen. Valuation of mortgage backed securities using Brownian Bridges to reduce effective dimension. J. Comp. Finance, 1:27-46, 1997.

http://www-stat. stanford.edu/ owen/reports/cmo.ps. C885

[2] R. Cont and P. Tankov. Financial Modelling with Jump Processes. Chapman \& Hall/CRC, Boca Raton, London, New York, Washington, D. C., 2004. C889

[3] P. Glasserman. Monte Carlo Methods in Financial Engineering. Springer, New York, Berlin, Heidelberg, Hong Kong, London, Milan, Paris, Tokyo, 2004. C886, C891, C894 
[4] S.G. Kou and H. Wang. Option pricing under a double exponential jump diffusion model. Management Science, 50:1178-1192, 2004. C885, C886, C888, C895

[5] A. Kyprianou, W. Schoutens, and P. Wilmott. Exotic option pricing and advanced Lévy models. Wiley, Chichester, 2005. C896

[6] P. L'Ecuyer and C. Lemieux. Recent advances in randomized quasi-Monte Carlo methods. In Modeling uncertainty, volume 46 of Internat. Ser. Oper. Res. Management Sci., pages 419-474. Kluwer Acad. Publ., Boston, MA, 2002. http:

//www.iro.umontreal.ca/ lecuyer/myftp/papers/survey01.ps. C886, C892

[7] S. M. Ross. Introduction to probability models. Harcourt/Academic Press, Burlington, MA, sixth edition, 1997. C890

\section{Author address}

1. Jan Baldeaux, Department of Mathematics and Statistics, University of New South Wales, Sydney, Australia. mailto: Jan. Baldeaux@student. unsw . edu . au 